Supporting Information

\title{
Optimization and Scale-Up of the Continuous Flow Acetylation and Nitration of 4-Fluoro-2-methoxyaniline to Prepare a Key Building Block of Osimertinib
}

\footnotetext{
Manuel Köckinger, ${ }^{\dagger \neq}$ Benjamin Wyler, $₫$ Christof Aellig, ${ }^{\S}$ Dominique M. Roberge,, Christopher A. Hone, $*,+, \uparrow$ and C. Oliver Kappe ${ }^{*,+, \ddagger}$

${ }^{\dagger}$ Center for Continuous Flow Synthesis and Processing (CCFLOW), Research Center Pharmaceutical Engineering GmbH (RCPE), Inffeldgasse 13, 8010 Graz, Austria

$\$$ Institute of Chemistry, University of Graz, NAWI Graz, Heinrichstrasse 28, A-8010 Graz, Austria § Microreactor Technology, Lonza AG, CH-3930 Visp, Switzerland
}

\section{Contents}

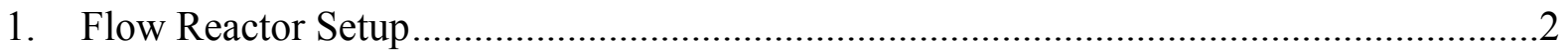

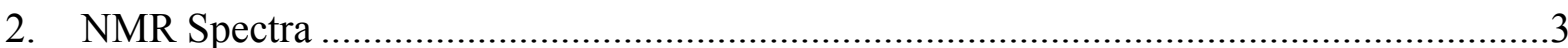




\section{Flow Reactor Setup}

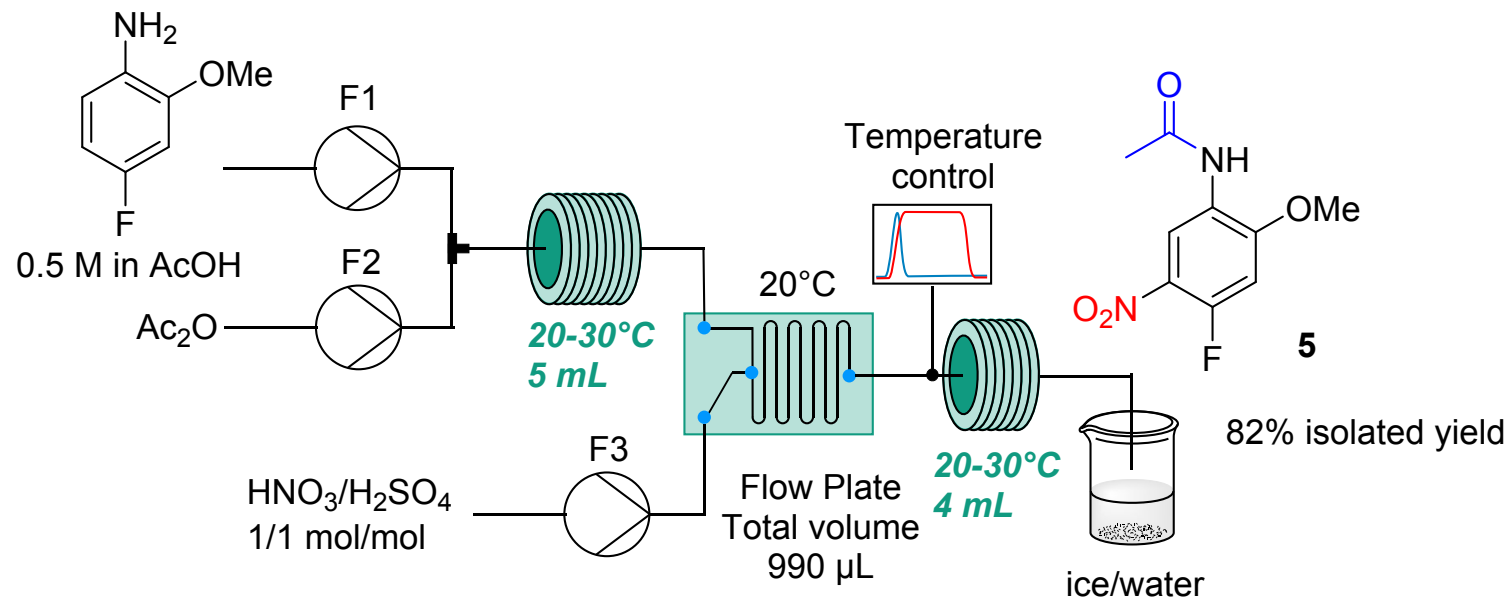

Figure S1. Continuous-flow setup (schematic) for the telescoped reaction.

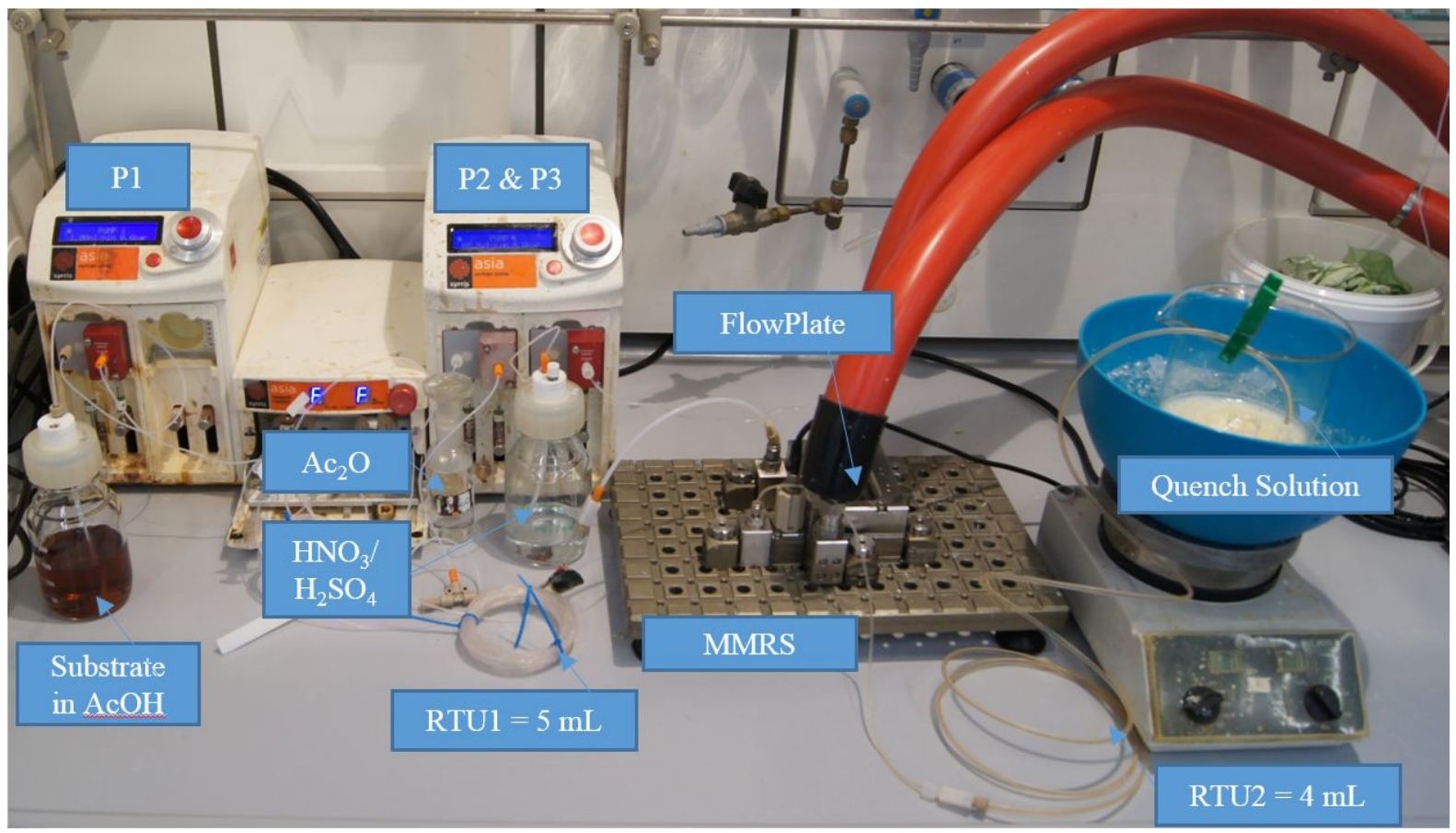

Figure S2. Labelled image of continuous-flow setup for the telescoped reaction. 


\section{NMR Spectra}

4-Fluoro-2-methoxy-5-nitroaniline

12.12.2018 10:22:14

\begin{tabular}{|c|c|c|c|c|c|c|c|}
\hline \multicolumn{8}{|c|}{\begin{tabular}{l|ll} 
Multiplets Integrals Sum 7.51 & Number of Nuclei & $8 \mathrm{H}$ 's \\
\end{tabular}} \\
\hline Acquisition Time (sec) & 3.9846 & Comment & 4-Fluoro-2 & xyy-5-nitroaniline PROTON & 25 S_LO DMSO C & BrukerITOPSPINIdat & antenbei 30 \\
\hline$D$ & 0.0002 & $D 1$ & 25 & $D E$ & 6.5 & DS & 2 \\
\hline Date & \multicolumn{3}{|c|}{$12 \operatorname{Dec} 2018$ 10:14:34 } & Date Stamp & \multicolumn{3}{|c|}{$12 \operatorname{Dec} 2018$ 10:14:34 } \\
\hline File Name & \multicolumn{7}{|c|}{ 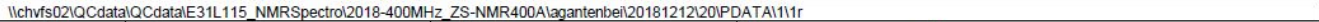 } \\
\hline Frequency $(\mathrm{MHz})$ & 400.130 & $G B$ & 0 & INSTRUM & $\langle$ spect $>$ & & 0.3 \\
\hline NS & 16 & Nucleus & $1 \mathrm{H}$ & Number of Transients & 16 & Origin & spect \\
\hline Original Points Count & 32768 & Owner & chv_nmr & & 1 & PROBHD & $<Z 108618$ 0439 (PA BBO 400S1 BBF-H-D-05 Z PLUS)> \\
\hline \begin{tabular}{|l|} 
PULPROG \\
\end{tabular} & $<2030>$ & Points Count & 65536 & Pulse Sequence & 2030 & Receiver Gain & 108.61 \\
\hline SF & \multicolumn{3}{|c|}{400.129995418733} & SFO1 & 400.132470967 & & 65536 \\
\hline SSB & 0 & SW(crclical) (Hz) & 8223.68 & SWH & \multicolumn{3}{|c|}{8223.68421052632} \\
\hline \begin{tabular}{|l} 
Solvent \\
\end{tabular} & DMSO-C & Spectrum Offset $(\mathrm{Hz})$ & 2475.5503 & Spectrum Type & standard & Sweep Width $(\mathrm{Hz})$ & 8223.56 \\
\hline TD & 65536 & & 1 & & 299.998 & Temperature /degre & 26.998 \\
\hline UNC1 & $\langle 1 \mathrm{H}>$ & WOW & 1 & & & & \\
\hline
\end{tabular}

User Notes 4-Fluoro-2-methoxy-5-nitroaniline.

PROTON 25s LO DMSO C: BrrukerlTOPSPINIdata agantenbei 30
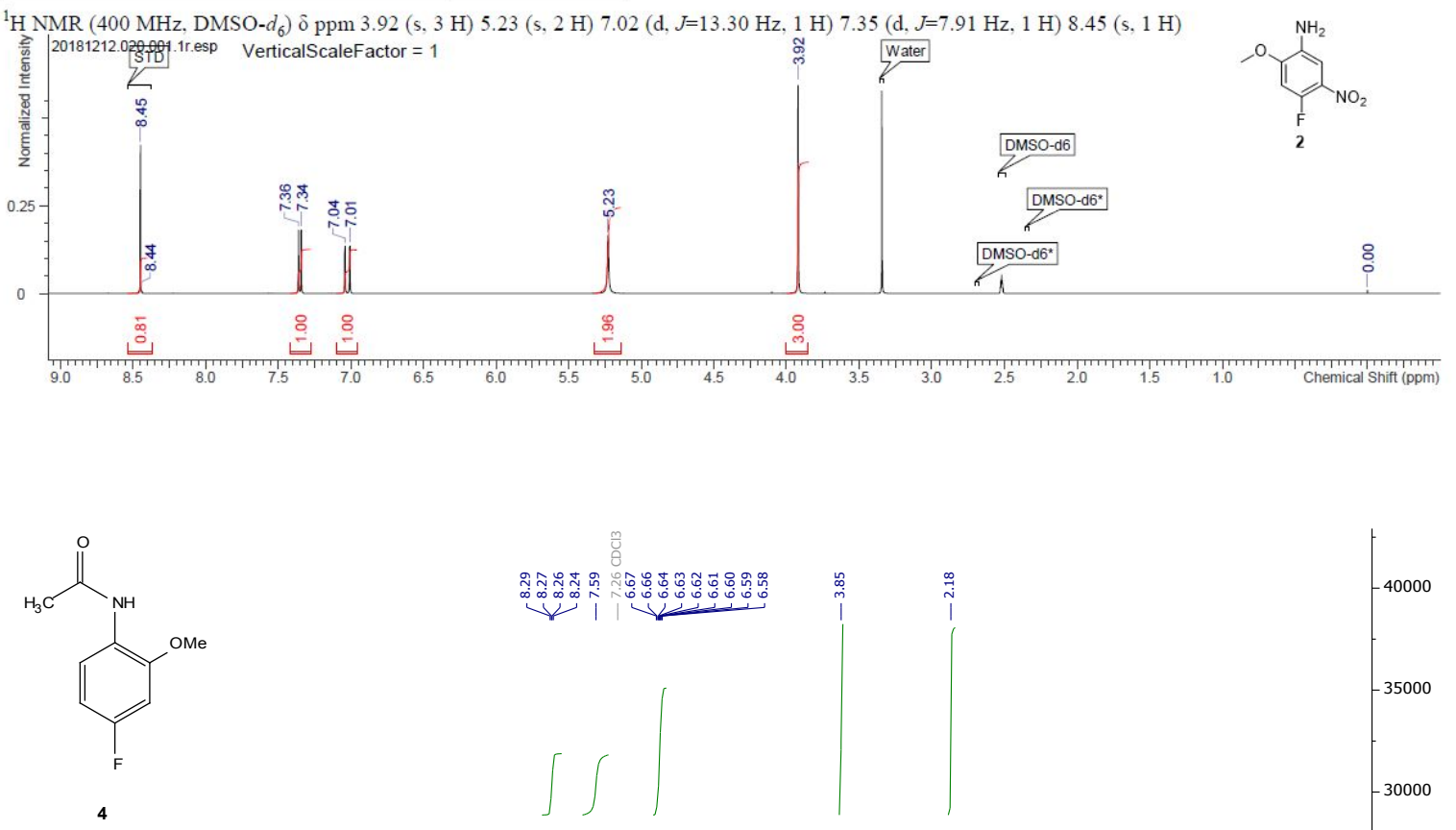

$-40000$

35000

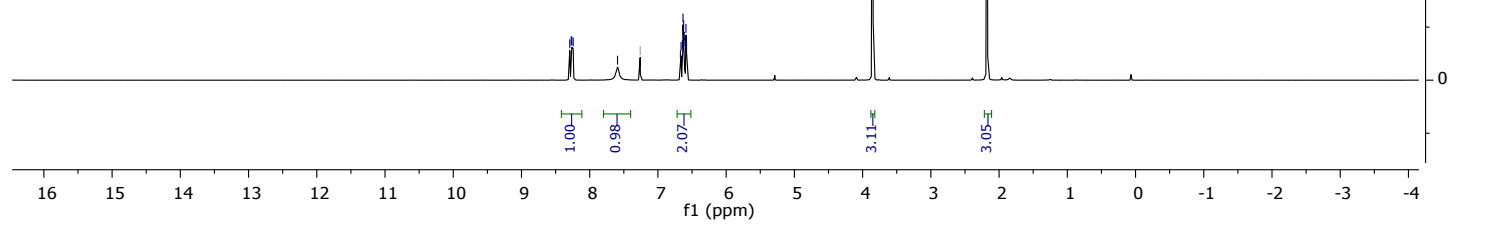




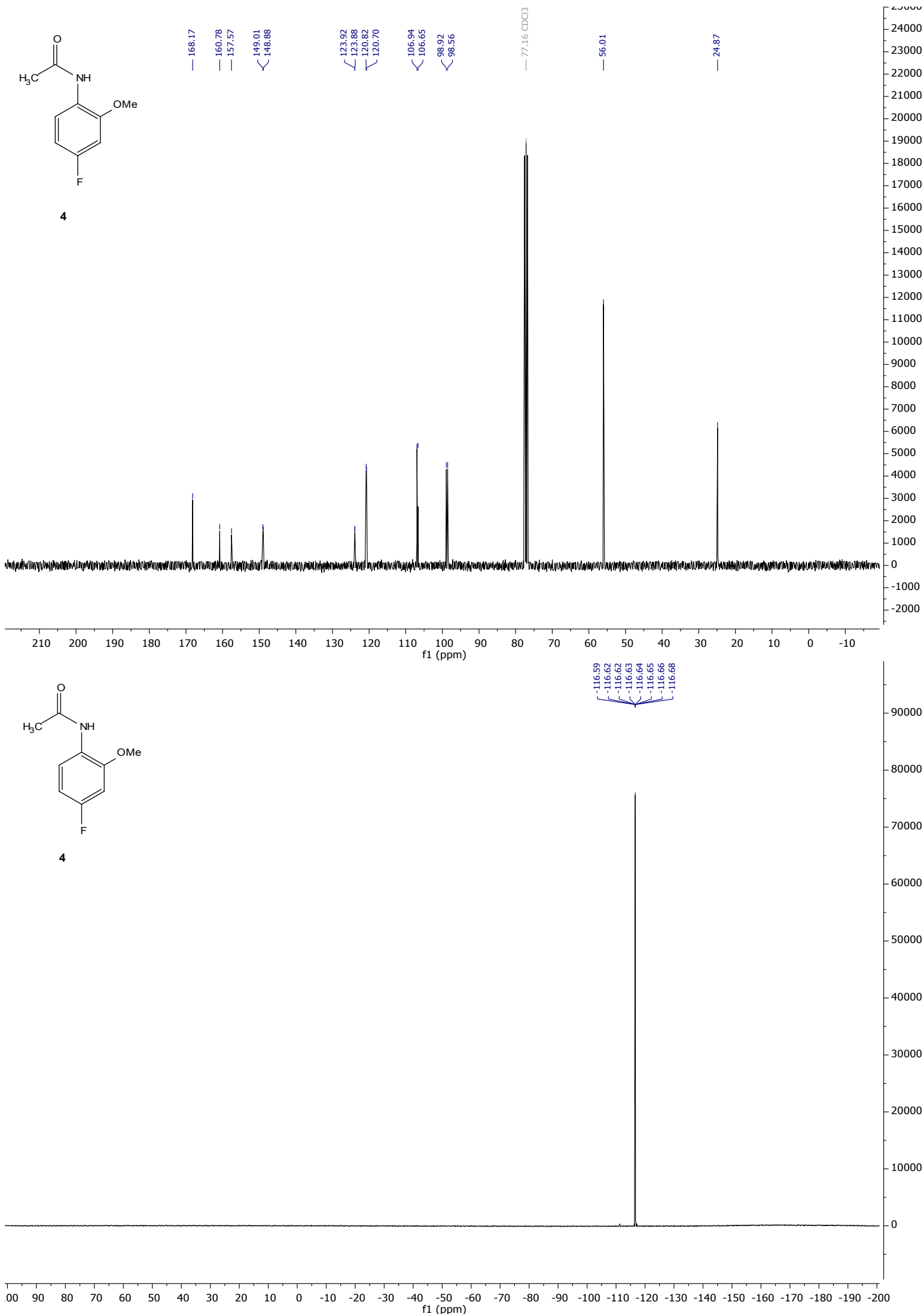



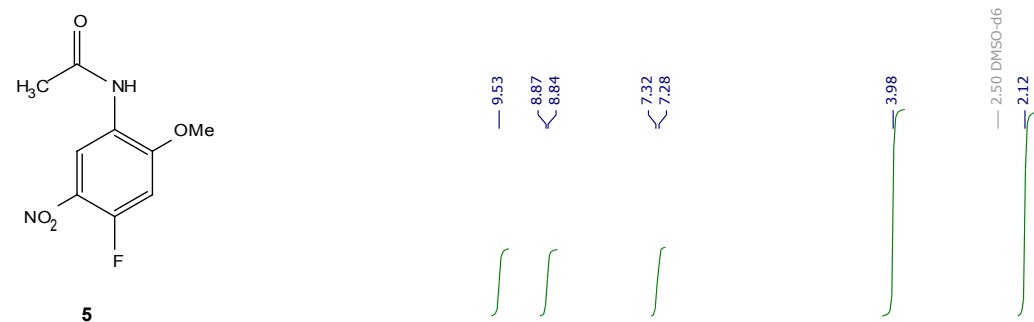

5500

5000

4500

4000

3500

3000

2500

2000

1500

000

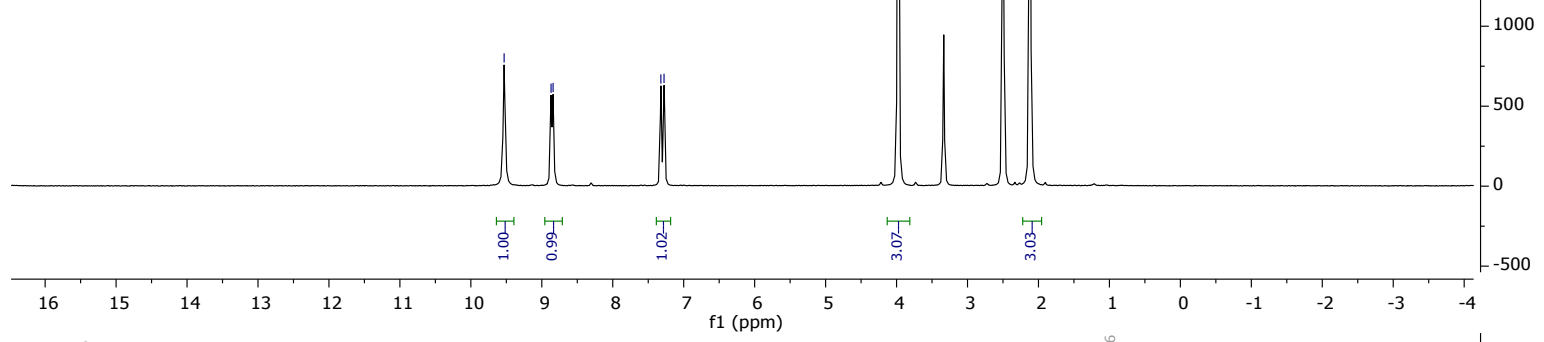

OMe

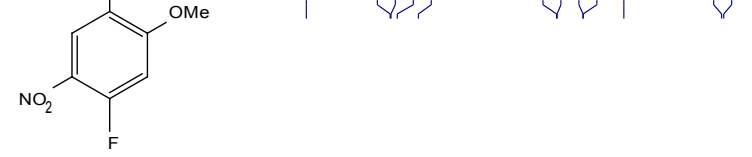

5

票

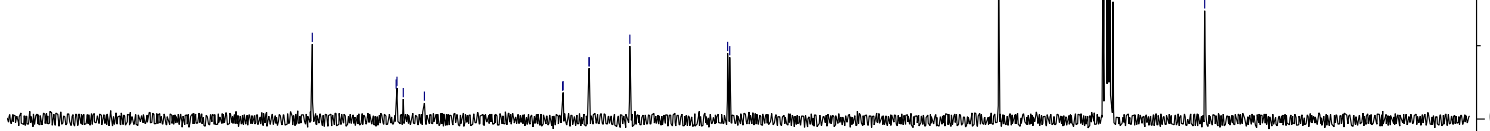

$\begin{array}{lllllllllll}210 & 200 & 190 & 180 & 170 & 160 & 150 & 140 & 130 & 120 & 110 \\ \mathrm{f} 1(\mathrm{ppm}) & 100\end{array}$ 

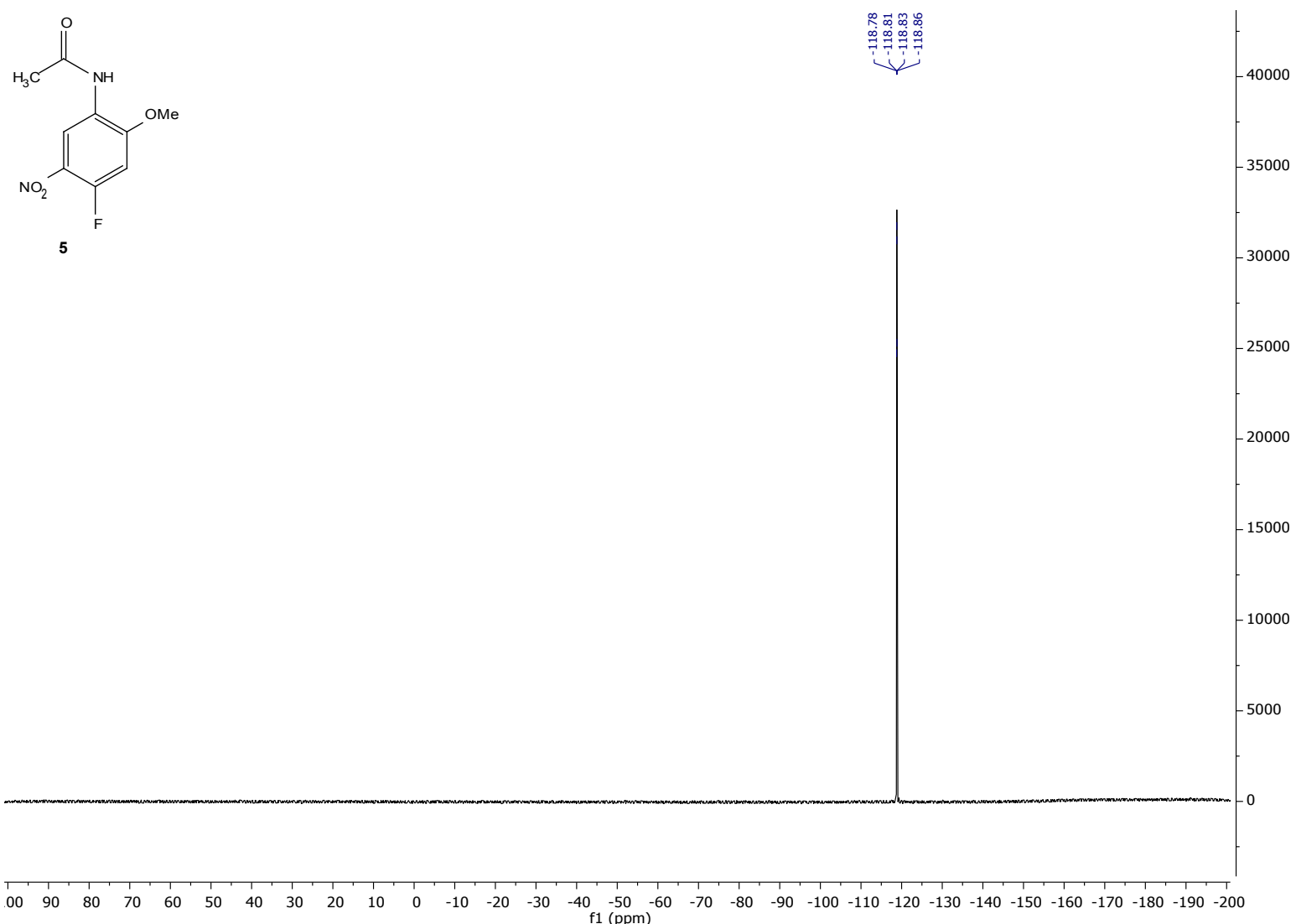

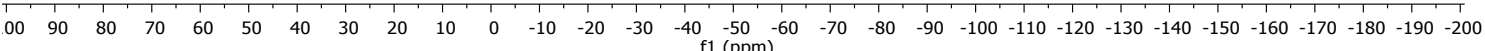

